\title{
Balance in Transitional Age Youth with Autism Spectrum and Neurodevelopmental Disorders during a 6-Week Comprehensive Wellness Program
}

\author{
Eve Spratt, MD, MSCR ${ }^{1 *}$, Carrie Papa ${ }^{1}$, Conner McDonald, COM², Martina Mueller, PhD ${ }^{3}$, Nicole \\ Gribben, MPH-PAPH, PhD Student ${ }^{4}$, Carolyn Peterseim, COM3², Mary Ashley Mercer, MD ${ }^{1}$, Ali Serpe, \\ $M D^{5}$, Tatiana Baier, $P D^{6}$ and Janis Newton ${ }^{6}$
}

\author{
${ }^{1}$ Division of Developmental-Behavioral Pediatrics, Medical University of South Carolina, USA \\ ${ }^{2}$ College of Medicine, Medical University of South Carolina, USA \\ ${ }^{3}$ College of Nursing, Medical University of South Carolina, USA \\ ${ }^{4}$ Arnold School of Public Health, University of South Carolina, USA \\ ${ }^{5}$ Department of Emergency Medicine, Indiana University School of Medicine, USA \\ ${ }^{6}$ Wellness Center, Medical University of South Carolina, USA
}

*Corresponding author: Eve Spratt, MD, MSCR, Professor of Pediatrics and Psychiatry, Director, Pediatric Psychiatry, Division of Developmental Pediatrics, Co-Director Piece it Together and Brain Health, Physical Health, Telehealth, Medical University of South Carolina, USA, Tel: 843-876-0444

\begin{abstract}
Young adults with Autism Spectrum Disorder (ASD) are at an increased risk of poor balance and coordination due to sensory deficits. Specifically, reactive postural control impairments have been well documented among individual's with ASD, however, research suggests that interventions can improve these associated deficiencies. The Piece It Together (PIT) program is a community-based comprehensive program designed to provide wellness opportunities among teens and young adults with ASD and other mild Neurodevelopmental Deficits (ND). The PIT curriculum includes exercise, nutrition, stress management, and socialization with a focus on strength and endurance training geared towards improving everyday functioning through the increase of awareness and participation in daily healthy lifestyle behaviors. Twenty participants, aged 14-29, attended 90-minute classes at the MUSC Wellness Center twice a week for six weeks and were encouraged to increase their physical activity daily. The mini-Balance Evaluations Systems Test (miniBEST) was administered during the first and last class session to assess balance changes among all study participants over the 6-weeks. Results showed significantly improved balance scores at the end of the program, from 23.9 to $25.2(p=0.028)$. Overall, the 6-week PIT program successfully improved balance and coordination in teens and young adults with ASD or mild ND.
\end{abstract}

\section{Introduction}

Autism Spectrum Disorder (ASD) is an increasingly prevalent disorder affecting one out of every fifty-nine children in the United States according to the CDC [1]. ASD is a neurodevelopmental disorder characterized by restricted, repetitive patterns of behavior and deficits in communication and social interactions [2]. Youth with ASD are at least as likely and possibly more likely to be obese than Typically Developing (TD) peers [3-5]. In addition to typical risk factors, individuals with ASD have additional challenges placing them at risk for obesity such as higher rates of selective or "picky" eating, which may be due to sensory disturbances, and report less variety in their diet [5-8]. Youth with ASD also exhibit more sedentary behavior and less physical activity than TD youth $[9,10]$. This sedentary behavior is linked to not only obesity and chronic health conditions but also mental health conditions such as depression [11]. Many individuals with ASD and those with other Neurodevelopmental Disorders (NDD) use psychopharmacological treatments that may contribute to weight gain 
[5]. In general, youth that are obese are very likely to continue being obese into adulthood which leads to an increased likelihood of many chronic health conditions [12]. Adults with ASD are more likely than the general population to have many medical issues such as diabetes, hypertension, dyslipidemia, and obesity [13]. Obesity also increases the likelihood of social and emotional consequences such as bullying and self-esteem issues [12]. Current research suggests youth with ASD are already at an increased likelihood for these social and emotional issues [14]. Adolescents and young adults are at a critical transitional age where early intervention can jumpstart healthy lifestyle habits. There is a paucity of such programs available to serve this population [15].

In the general population, exercise interventions have been shown to be effective in addressing numerous mental health issues such as depression and anxiety $[16,17]$, disorders more common in individuals with ASD [18], and also boost cognitive performance $[19,20]$. However, it is important to consider that the typical interventions that are effective for the general population may not be effective for the ASD population. In particular, youth with ASD face many different barriers and additional risk factors than TD youth in addressing these issues with interventions. Barriers to physical activity for this population include lack of interest, lack of appropriate programs, and behavioral issues [21]. Youth with ASD also find physical activities harder to learn and this is partially due to balance and coordination concerns that may co-exist with ASD and NDD. Fewer youth with ASD and NDD believe that wellness programs are a way to make friends than TD youth [22]. Often, youth with ASD prefer not to exercise or exercise at home rather than exercise with peers [23]. Parents of children with ASD cite various reasons for their children not participating in physical activities, which include the lack of appropriate skills in other adults who interact with their children, as well as their children having few friends and often being excluded by their peers. Each of these responses was significantly inversely correlated with the child's amount of physical activity [24].

More importantly, while it is not currently included in the ASD diagnostic criteria, accumulating research has identified motor deficits as a comorbidity to ASD which may ultimately impair coordination, motor planning, and particularly balance among those with ASD [25-27]. These motor deficits may hinder social and emotional development, though more research is necessary to determine to what extent and if they contribute to social deficits in the ASD population [28]. Such motor deficits may disproportionally impair an individual with ASD's overall exercise capacity, and their perception and interest in exercise-related behavior. A physical impairment in one's ability to properly execute, function and perform a successful exercise-related movement as a result of their inability to balance may psychologically control a large degree of their overall attitude and feelings of self-efficacy toward physical activity. In other words, the inability to balance among those with ASD may subconsciously alter their initial readiness, empowerment, confidence, and therefore, their willingness to engage in exercise. If individuals with ASD are more likely to avoid behaviors that require them to balance, this may subconsciously drive them to show an increase in interest and participation for behaviors that are primarily sedentary and less physical in nature. This may explain the alarming rates of sedentary behavior, lack of physical activity, and increase in obesity and chronic disease risk that appear more evident among the ASD population. Preliminary research suggests that exercise interventions can not only be beneficial in improving motor issues, but also beneficial in improving social deficits and behavioral problems [29-31]. Additionally, interventions of physical therapists to improve these motor deficits is promising [32]. However, more research is necessary to determine the best protocol for intervention programs to address the aforementioned issues in the ASD population. This Piece It Together research study hopes to illuminate some answers to this question.

Piece It Together is a comprehensive wellness intervention program devised to address barriers to holistic wellness specific amongst the population of adolescents and young adults with ASD and other developmental disabilities. The Piece It Together multidisciplinary approach has been created through the collaboration of physicians, physical and occupational therapists, personal trainers, and registered dieticians and tailored to better serve the ASD population. Based on the target population, Piece It Together focuses on five main pillars: exercise, nutrition, socialization, stress relief, and getting out of your comfort zone. Effort is focused on promoting the long-term adoption of healthy lifestyle habits. Additional reports have discussed the effectiveness of the PIT intervention in improving body composition, physical fitness, exercise habits, eating habits, mood, and will discuss success, challenges, and future directions to assist similar programs around the nation. While additional outcomes such as strength, flexibility, depression and anxiety, were collected as part of the larger PIT program, the purpose of this pilot study was to assess balance over the course of the 6-week wellness program.

\section{Method}

Twenty participants, ages 14-28 (average age 19.7, 11 male and 9 female), diagnosed with ASD or another developmental disorder enrolled and completed the Piece it Together Summer Program, consisting of one and a half hour long classes, twice a week, for six weeks. Pre-testing was performed before the start of the program, and post-testing was performed at the end of the program. The purpose of this study was to 
assess balance as measured by the mini-Balance Evaluations Systems Test (miniBEST) [33,34] before and after a fitness intervention in youth with ASD or other NDD. Additional testing consisted of InBody 570 Body Composition Analyzer@2014 for body composition, a timed run for cardiovascular fitness, the miniBEST for balance evaluation, the Patient Health Questionnaire 9 (PHQ9), Beck Anxiety Index for Youth (BAI), and the 5-2-1-0 Healthy Habits Questionnaire. These assessments were performed to track quantitative and qualitative changes in health components related to physical (i.e. body composition, fitness, balance), as well as lifestyle and mood factors over the duration of the PIT intervention, that have been discussed elsewhere.

\section{Intervention Overview}

The general format of the Piece it Together classes $[35,36]$ consisted of a teaching lesson, followed by 30 45 minutes of moderate to vigorous exercise, and ending with stress relief practice. Class content varied each session. Exercise typically consisted of a warmup, which included stretching and basic balance activities such as tandem walking and balancing on one leg, followed by exercise classes that included spin, water aerobics, cardio circuits, soccer, Tae Bo, and boxing led by personal trainers. Participants were encouraged to exercise outside of class time as well but this tended to be minimal. Nutrition discussions were led by a registered dietician and focused on eating more fruits and vegetables, appropriate portion sizes, and increasing water intake. A trip was made to the nearby Medical University Urban Farm to further educate participants and healthy foods were brought to class to encourage trying new foods. Stress relief techniques consisted of yoga, meditation, visualization, and relaxation and breathing exercises. Socialization was encouraged through active participation in class discussions and interactions with other peers, instructors, and volunteers throughout the program. Participants who had previously participated in the program were encouraged to be peer leaders and promote program advocacy.

\section{Balance Evaluation}

Testing was completed at baseline (pre-intervention) and after 6 weeks (post-intervention). The miniBEST assesses balance and provides a total score and sub-scores for different types of balance systems. This instrument was chosen as it assesses deficits that have been previously reported in this population [26,3740]. The miniBEST has shown good interrater reliability,

Table 1: Mean miniBEST Pre and Post Scores.

\begin{tabular}{|l|l|l|l|l|l|}
\hline & Pre-Score & Post-Score & Difference & 95\% Confidence interval & p-value $^{*}$ \\
\hline Reactive Postural Control (maximum =6) & $4.4 \pm 1.0$ & $5.1 \pm 0.7$ & $0.8 \pm 1.0$ & $0.3 ; 1.3$ & 0.011 \\
\hline Sensory Orientation (maximum =6) & $5.9 \pm 0.2$ & $5.5 \pm 0.7$ & $-0.4 \pm 0.6$ & $-0.8 ;-0.1$ & 0.016 \\
\hline Anticipatory (maximum = 6) & $5.4 \pm 0.9$ & $5.5 \pm 0.6$ & $0.1 \pm 0.6$ & $-0.2 ; 0.4$ & 0.688 \\
\hline Dynamic Gait (maximum = 10) & $8.5 \pm 1.1$ & $9.1 \pm 0.9$ & $0.6 \pm 1.3$ & $-0.1 ; 1.2$ & 0.097 \\
\hline Total Score (maximum = 28) & $23.9 \pm 2.4$ & $25.2 \pm 2.0$ & $1.2 \pm 2.2$ & $0.2 ; 2.3$ & 0.028 \\
\hline
\end{tabular}

*obtained from Wilcoxon Signed Rank test.

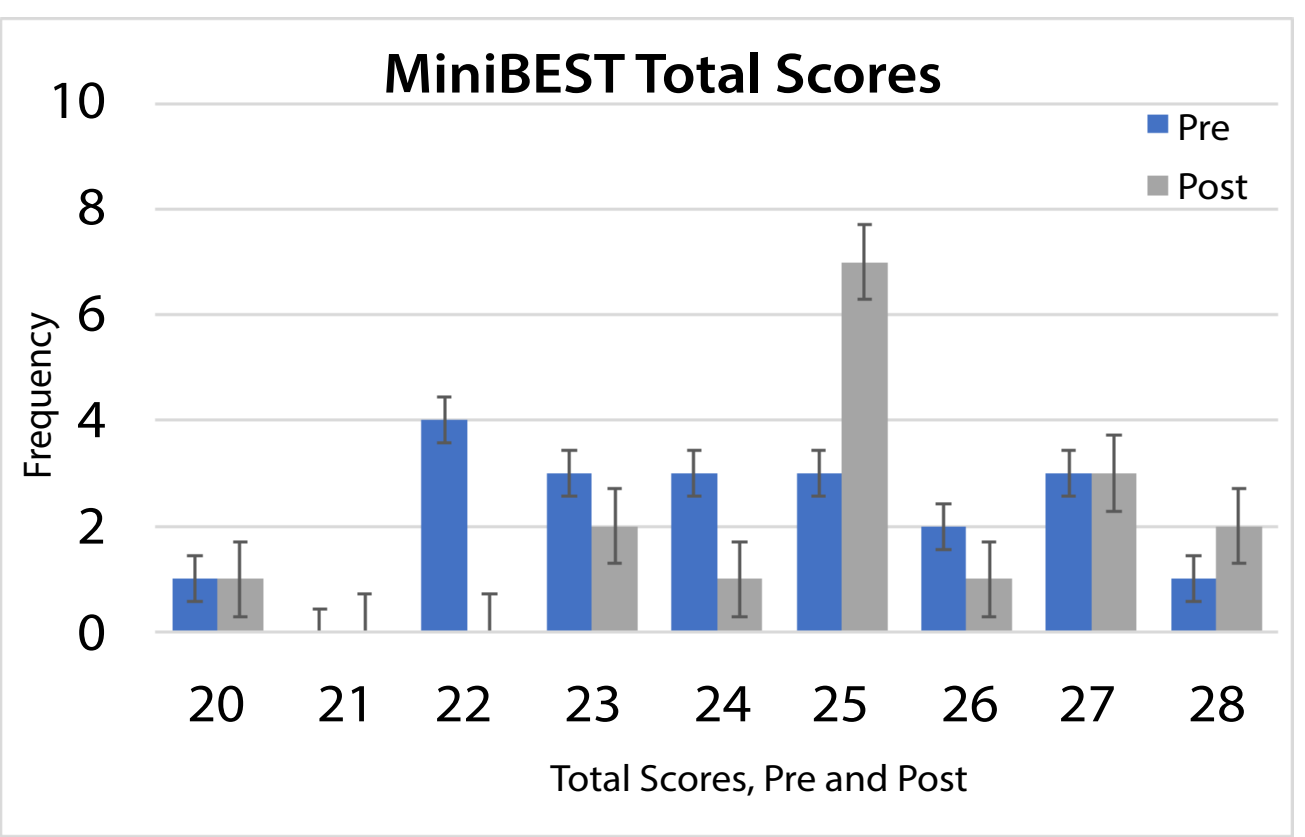

Figure 1: Total miniBEST Scores Pre and Post-testing by frequency. The miniBEST has a maximum total score of 28 . Scores in this cohort ranged from 20 to 28. 
test-retest reliability, correlation with other balance assessments, and validity in determining fall risk [3]. The miniBEST is scored on a scale from 0-2 on 14 balance skills that include: anticipatory balance, reactive postural control, sensory orientation, and dynamic gait. 0 is physically unable to perform the action, 1 is performing the action with some imbalance, and 2 is performing the action without difficulties. The maximum total score one can achieve on the miniBEST is a 28 per test. The assessment was scored by an investigator and provided a reliable and valid assessment of balance in the general population [34].

\section{Results}

In the Piece it Together Summer Program, 20 participants (11 males; mean age 19.7 years) consented to the Institutional Review Board (IRB) approved research study. Parents and participants self-reported medical diagnoses. Study subjects were diagnosed with one or more neuropsychiatric diagnoses including ASD, ADHD and other mild Intellectual Disabilities (ID). Sixteen of the subjects were diagnosed with ASD and two were diagnosed with mild or moderate ID. Total and subscales scores on the miniBEST obtained pre-intervention and post-intervention were compared using Wilcoxon Signed Rank tests. The mean total miniBEST score increased from 23.9 to 25.2 ( $p=0.028$ ) indicating improved balance (Table 1 and Figure 1 ). Mean scores for dynamic gait, anticipatory and reactive postural control categories trended towards improving, but were not significant. Sensory orientation means scores decreased at post-test.

\section{Discussion}

This pilot study demonstrated the presence of balance deficits in this population. The MiniBEST normative data for older populations has shown healthy controls score no less than $26[3,4]$. The mean from this population of ASD and NDD individual's prior to intervention was 23.9, indicating there may be balance deficits present in this population. Scores did improve after completion of the 6-week PIT program suggesting that balance deficits for youth with ASD or NDD may improve with brief community-based group interventions.

An additional goal of the Piece It Together program is to provide others with the tools, of which were devised and utilized by the program itself, to implement similar interventions. There were several challenges in implementing the intervention program. These included behavioral problems, lack of participants' motivation, and selecting exercises that were appropriate for such a heterogeneous group. There were some limitations to this study. Reliability of raters was not tested, and raters were not blinded. The 6-week program duration may be too short a period to examine substantial impacts, but the trend is encouraging. Balance was not the primary focus of the intervention, therefore the program did not primarily consist of balance training, further, higher post-test values may be a reflection of participant learning and familiarity rather than a result of the intervention. While no ceiling effect was seen in previous studies with the miniBEST, those studies had lower mean scores [3]. Use of a different balance outcome that could better capture detailed balance information could display more significant improvement. There was no long-term follow up to determine if habits continued after the end of the program.

Overall, the presence of a significant balance deficit seen in our study on the miniBEST suggests that typical exercise and coordination interventions are indicated as a part of a comprehensive wellness intervention plan as these deficits can improve, but can present as a barrier to physical activity. This research supports that motor deficits may impair coordination, motor planning, and particularly balance among those with ASD. While it is not currently included in the ASD diagnostic criteria, motor deficits as a comorbidity to ASD may be supported by the findings in this study, and health care professionals are encouraged to consider these. Future research aims involving similar participants and interventions should include identification of the mechanism attributing to balance impairment, determining which targeted activities within the intervention would be most effective for enhancing balance, and clearly analyzing and defining gait coordination deficits, and gait analyses.

\section{Acknowledgements}

Funding and volunteer support provided by the following: NIH/NCATS UL1TR000062, The Charles Webb/ Ed Croft Trust Endowment of Coastal Community Foundation, The Coastal Community Foundation Cooper River Bridge Run Scholarship Grants, SC Developmental Disabilities Council Grants 05-21-0005, 06-21-0005, and 07-21-0005 (2015-2018), South Carolina Translational Research Community Engagement Grant (2014), Crews Subaru of Charleston, and the Lowcountry Autism Foundation (501c3). Thank you to Physical Therapy consultant Cynthia Dodds, and her student, Caitlin Keller. Many thanks to all the families, participants, volunteers and other donors for their support!

\section{References}

1. Baio J WL, Wiggins L, Christensen DL, Maenner MJ, Daniels J, et al. (2018) Prevalence of Autism Spectrum Disorder Among Children Aged 8 Years - Autism and Developmental Disabilities Monitoring Network, 11 Sites, United States, 2014. MMWR Surveill Summ 67: 1-23.

2. American Psychiatric Association (2013) Diagnostic and Statistical Manual of Mental Disorders. ( $5^{\text {th }}$ edn), Washington DC: American Psychiatric Publishing.

3. Egan AM, Dreyer ML, Odar CC, Beckwith M, Garrison CB (2013) Obesity in young children with autism spectrum disorders: prevalence and associated factors. Child Obes (Print) 9: 125-131.

4. Bandini LG, Curtin C, Hamad C, Tybor DJ, Must A (2005) 
Prevalence of Overweight in Children with Developmental Disorders in the Continuous National Health and Nutrition Examination Survey (NHANES) 1999-2002. J Pediatr 146: 738-743.

5. Curtin C, Jojic M, Bandini LG (2014) Obesity in children with autism spectrum disorder. Har Rev Psychiatry 22: 93-103.

6. Sharp WG, Berry RC, McCracken C, Nuhu NN, Marvel E, et al. (2013) Feeding Problems and Nutrient Intake in Children with Autism Spectrum Disorders: A Meta-analysis and Comprehensive Review of the Literature. J Autism Dev Disord 43: 2159-2173.

7. Tavassoli T, Baron-Cohen S (2012) Taste Identification in Adults with Autism Spectrum Conditions. J Autism Dev Disord 42: 1419-1424.

8. Schreck KA, Williams K, Smith AF (2004) A comparison of eating behaviors between children with and without autism. J Autism Dev Disord 34: 433-438.

9. Jones RA, Downing K, Rinehart NJ, Barnett LM, May T, et al. (2017) Physical activity, sedentary behavior and their correlates in children with Autism Spectrum Disorder: A systematic review. PLoS ONE 12: 0172482.

10. Must A, Phillips SM, Curtin C, Anderson SE, Maslin M, et al. (2014) Comparison of sedentary behaviors between children with autism spectrum disorders and typically developing children. Autism 18: 376-384.

11. Zhai L, Zhang Y, Zhang D (2015) Sedentary behaviour and the risk of depression: a meta-analysis. Br J Sports Med 49: 705-709.

12. Must A, Strauss RS (1999) Risks and consequences of childhood and adolescent obesity. Int $\mathrm{J}$ Obes Relat Metab Disord 23: 2-11.

13. Croen LA, Zerbo O, Qian Y, Massolo ML, Rich S, et al. (2015) The health status of adults on the autism spectrum. Autism 19: 814-823.

14. Cappadocia MC, Weiss JA, Pepler D (2012) Bullying experiences among children and youth with autism spectrum disorders. J Autism Dev Disord 42: 266-277.

15. Goldschmidt J, Song HJ (2015) At-risk and underserved: a proposed role for nutrition in the adult trajectory of autism. $\mathrm{J}$ Acad Nutr Diet 115: 1041-1047.

16. Blumenthal JA, Babyak MA, Doraiswamy PM, Watkins L, Hoffman BM, et al. (2007) Exercise and pharmacotherapy in the treatment of major depressive disorder. Psychosom Med 69: 587-596.

17. Asmundson GJ, Fetzner MG, Deboer LB, Powers MB, Otto MW, et al. (2013) Let's get physical: a contemporary review of the anxiolytic effects of exercise for anxiety and its disorders. Depress Anxiety 30: 362-373.

18. Strang JF, Kenworthy L, Daniolos P, Case L, Wills MC, et al. (2012) Depression and Anxiety Symptoms in Children and Adolescents with Autism Spectrum Disorders without Intellectual Disability. Res Autism Spectr Disord 6: 406-412.

19. Chang YK, Labban JD, Gapin JI, Etnier JL (2012) The effects of acute exercise on cognitive performance: A metaanalysis. Brain Res 1453: 87-101.

20. Fedewa AL, Ahn S (2011) The effects of physical activity and physical fitness on children's achievement and cognitive outcomes: a meta-analysis. Res Q Exerc Sport 82: $521-535$.

21. Yazdani S, Yee CT, Chung PJ (2013) Factors Predicting Physical Activity Among Children With Special Needs. Prev
Chronic Dis 10: 119.

22. Stanish H, Curtin C, Must A, Phillips S, Maslin M, et al. (2015) Enjoyment, Barriers, and Beliefs About Physical Activity in Adolescents with and without Autism Spectrum Disorder. Adapt Phys Activ Q 32: 302-317.

23. Sankovich $L$ (2013) Development and Implementation of a Video-Based Physical Activity Preference Assessment for Children with Autism and Their Parents. ProQuest LLC.

24. Must A, Phillips S, Curtin C, Bandini LG (2015) Barriers to physical activity in children with autism spectrum disorders: Relationship to physical activity and screen time. J Phys Act Health 12: 529-534.

25. Downey R, Rapport MJ (2012) Motor Activity in Children with Autism: A Review of Current Literature. Pediatr Phys Ther 24: 2-20.

26. Fournier KA, Hass CJ, Naik SK, Lodha N, Cauraugh JH (2010) Motor Coordination in Autism Spectrum Disorders: A Synthesis and Meta-Analysis. J Autism Dev Disord 40: 1227-1240.

27. Matson ML, Matson JL, Beighley JS (2011) Comorbidity of physical and motor problems in children with autism. Res Dev Disabil 32: 2304-2308.

28. Leonard HC (2016) The Impact of Poor Motor Skills on Perceptual, Social and Cognitive Development: The Case of Developmental Coordination Disorder. Front Psychol 7: 311.

29. Ketcheson L, Hauck J, Ulrich D (2017) The effects of an early motor skill intervention on motor skills, levels of physical activity, and socialization in young children with autism spectrum disorder: A pilot study. Autism 21: 481492.

30. Sowa M, Meulenbroek R (2012) Effects of physical exercise on Autism Spectrum Disorders: A meta-analysis. Research in Autism Spectrum Disorders 6: 46-57.

31. Sotoodeh MS, Arabameri E, Panahibakhsh M, Kheiroddin $\mathrm{F}$, Mirdoozandeh $\mathrm{H}$, et al. (2017) Effectiveness of yoga training program on the severity of autism. Complement Ther Clin Pract 28: 47-53.

32. Mieres AC, Kirby RS, Armstrong KH, Murphy TK, Grossman $L$ (2012) Autism spectrum disorder: an emerging opportunity for physical therapy. Pediatr Phys Ther 24: 31-37.

33. Tsang CS, Liao LR, Chung RC, Pang MY (2013) Psychometric Properties of the Mini-Balance Evaluation Systems Test (Mini-BESTest) in Community-Dwelling Individuals with Chronic Stroke. Phys Ther 93: 1102-1115.

34. Horak FB, Wrisley DM, Frank J (2009) The Balance Evaluation Systems Test (BESTest) to Differentiate Balance Deficits. Phys Ther 89: 484-498.

35. Spratt EG, Norton J, Papa C, Newton J, McDonald C, et al. (2019) "The PIT Experience": A young adult with Autism Spectrum Disorder's opinion of how a wellness program changed her life. J Autism Dev Disord 17.

36. SprattEG, Mercer M, Grimes A, Papa C, Norton J, etal. (2018) Translating benefits of exercise on depression for youth with autism spectrum disorder and neurodevelopmental disorders. J Psychol Psychiatr 2: 109.

37. Cheldavi H, Shakerian S, Shetab Boshehri SN, Zarghami $M$ (2014) The effects of balance training intervention on postural control of children with autism spectrum disorder: Role of sensory information. Research in Autism Spectrum Disorders 8: 8-14. 
38. Freitag CM, Kleser C, Schneider M, von Gontard A (2007) Quantitative Assessment of Neuromotor Function in Adolescents with High Functioning Autism and Asperger Syndrome. J Autism Dev Disord 37: 948-959.

39. Minshew NJ, Sung K, Jones BL, Furman JM (2004)
Underdevelopment of the postural control system in autism. Neurology 63: 2056-2061.

40. Molloy CA, Dietrich KN, Bhattacharya A (2003) Postural Stability in Children with Autism Spectrum Disorder. J Autism Dev Disord 33: 643-652. 